\title{
Transtorno afetivo bipolar na infância \\ e na adolescência
}

Bipolar disorder in childhood and adolescence

Lee Fu-I ${ }^{\mathrm{a}}$

${ }^{a}$ Ambulatório de Transtornos Afetivos do Serviço de Psiquiatria da Infância e da Adolescência do Instituto de Psiquiatria do Hospital das Clínicas da Faculdade de Medicina da Universidade de São Paulo (FMUSP)

\begin{abstract}
Resumo
Os conhecimentos sobre transtorno afetivo bipolar com início na infância e na adolescência têm avançado muito nos últimos 15 anos. Atualmente, os esforços estão dirigidos para investigar o quadro clínico, para o desenvolvimento de instrumentos que auxiliam diagnóstico precoce e investigações de melhores formas de tratamentos de crianças e adolescentes portadores do transtorno. O presente texto tem objetivo de apresentar as principais características clínicas do transtorno em crianças e adolescentes, assim como as denominações, as descrições de tipos clínicos e do padrão de ciclagem mais comum da doença em jovens. Discussões sobre comorbidades, diagnóstico diferencial e avanço em tratamento farmacológico também serão apresentados.
\end{abstract}

Descritores: Transtorno bipolar/diagnóstico; Transtorno bipolar/terapia; Criança; Adolescente

\begin{abstract}
Many advances in the knowledge of childhood- and adolescent-onset bipolar disorder have been seen over the last 15 years. Current efforts focus on investigating clinical features, developing more instruments for early diagnosis and improving treatment research. The present study aims to present the main clinical characteristic of the disorder in children and adolescents, as well as the nomenclature, description of clinical phenotypes and the most common cycling pattern in youths. A discussion of comorbidity, differential diagnosis and advances in psychopharmacological treatment will also be presented.
\end{abstract}

Keywords: Bipolar disorder/diagnosis; Bipolar disorder/therapy; Child; Adolescent

\section{Introdução}

Até 15 anos atrás, ainda se discutia sobre a possibilidade de ocorrência ou não de transtornos afetivos bipolar (TAB) em crianças. Hoje, a ocorrência do transtorno já foi constatada e as atenções estão voltadas para a investigação das apresentações clínicas de TAB com início na infância e na adolescência (TAB-IA), desenvolvimento de instrumentos para auxiliar no diagnóstico precoce e investigações de melhores formas de tratamentos. ${ }^{1-5}$

O reconhecimento de sintomas depressivos ou de (hipo)mania em crianças costuma ser difícil, principalmente porque estas podem ter dificuldade em reconhecer e nomear seus próprios sentimentos. A baixa prevalência também pode ser devido ao fato de que as características consideradas atípicas em adultos parecem ser regra e não exceção em crianças e, por isso, muitos profissionais nem chegam a incluir o TAB como possibilidade de diagnóstico quando avaliam uma criança. ${ }^{1,3,5-12}$

Sabe-se que dados epidemiológicos podem variar de acordo com a idade, com o curso da doença e com a presença ou não de comorbidades. ${ }^{13-14}$ Apesar de estudos epidemiológicos apontarem que o TAB afeta homem e mulher igualmente na fase adulta, parece ser mais comum em meninos do que em meninas. Faedda et al verificaram que, enquanto pré-púberes, os meninos têm aproximadamente quatro vezes maior chance de receber diagnóstico de TAB do que as meninas. ${ }^{1}$ Findling et al também encontraram uma proporção duas vezes maior de meninos sobre meninas na amostra do seu estudo. ${ }^{7}$

Tanto o DSM-IV ${ }^{13}$ como a CID-10 ${ }^{14}$ citam o fato de que os quadros clínicos podem ser diferentes de acordo com a faixa etária e sugerem sintomas equivalentes ou substitutivos para crianças e adolescentes, mas essas medidas não parecem ser suficientes para o diagnóstico de TAB-IA. ${ }^{15} \mathrm{Em}$ debate promovido por $\mathrm{NIMH}$, considerou-se que há, na prática clínica, dois tipos de crianças com possibilidade de TAB: o primeiro grupo seria de crianças que apresentam todos os sintomas e características exigidos por DSM-IV ${ }^{13}$ para diagnóstico de TAB tipo-I ou tipo-II, ${ }^{12}$ e o segundo grupo seria de crianças que apresentam apenas alguns sintomas de TAB, porém não os principais, e sofrem cronicamente de instabilidade de humor, com funcionamento global severamente comprometido. Por ter partes de características de TAB, este segundo geralmente recebe diagnóstico de TAB não especificado (TAB-NOS). ${ }^{12}$ O termo TAB-NOS foi considerado um bom diagnóstico operacional ("working diagnostic") para casos pouco definidos pela maioria dos pesquisadores. ${ }^{12}$

Para definição de diagnóstico aconselha-se que as crianças tenham seus quadros clínicos bem descritos, com avaliação cuidadosa e sistematizada de todos os sintomas, verificando a freqüência de ocorrência e o grau de comprometimento de cada sintoma. Atualmente, recomenda-se os seguintes instrumentos de apoio a diagnóstico para TAB-IA: ${ }^{12}$ K-SADS versão PL (Schedule for Affective Disorders and Schizophrenia for SchoolAge Children-Present and Lifetime Version), ${ }^{16} \mathrm{~K}-\mathrm{SADS}$ com seção de transtorno afetivo e de ciclagem rápida de WASH-U$\mathrm{KSADS}^{17}$ e Washington University in St. Louis Kiddie Schedule for Affective Disorders and Schizophrenia (WASH-U-K-SADS). ${ }^{17}$ Note-se que a versão brasileira de K-SADS-PL já foi validada e se encontra disponível no nosso meio. ${ }^{18}$ Um outro instrumento semiestruturado para exploração de sintomas e sinais independente do diagnóstico é o CBCL (Child Behavior Checklist). ${ }^{19}$ Esse instrumento tem sido empregado em recentes pesquisas e mantém a sua importância nos estudos de TAB-IA. ${ }^{20} \mathrm{~A}$ versão brasileira deste também já se encontra disponível no nosso meio. ${ }^{21}$

\section{Aspecto clínico}

As crianças com TAB podem ser vistas como irritadas mesmo nos momentos de eutimia e a maioria merece diagnóstico de transtorno oposicional-desafiante mesmo em eutimia. ${ }^{7}$ Alguns pes- 
quisadores também interpretaram os sintomas de ansiedade das crianças enquanto eutímico como sinal de estado de humor persistentemente anormal associado ao TAB-IA. ${ }^{7} E$ freqüente o TAB de início precoce ter como a primeira manifestação do transtorno um episódio de depressão. Estudos mostraram que aproximadamente metade das crianças portadoras de TAB já apresentaram quadros de depressão anterior ao primeiro episódio de (hipo)mania. ${ }^{2,7}$ Geller et al, por sua vez, observaram que boa parte das crianças (32\%) em tratamento por depressão desenvolveram sintomas de (hipo)mania em quatro anos de seguimento. ${ }^{22}$

As características do episódio depressivo em crianças com TABIA são: início muito precoce (menor do que 13 anos); presença de retardo psicomotor alternando com agitação; presença de sintomas psicóticos; reações de (hipo)mania após uso de antidepressivo, hipersonia e hiperfagia. ${ }^{1-2,23}$ Estas características também são considerados sinais preditivos e de risco para posterior aparecimento de episódio de (hipo)mania em crianças deprimidas. ${ }^{1-2}$ Assim como em adultos, a ocorrência de tentativas de suicídio também é maior em TAB-|A. 3,15,23

Crianças em crise de (hipo)mania podem apresentar, além do humor exaltado e euforia, também, ou somente, aumento de irritabilidade e humor instável. Podem se auto-agredir e ser agressivas com outros, ${ }^{8,23}$ sendo freqüentes os relatos de criançasmodelo que, subitamente, tornaram-se "selvagens como bichos". 9,24 As crianças ficam hiperativas, falam muito mais e mais rápido do que o costume e apresentam aumento de distratibilidade. A atitude é de inquietação e de excitação constante e com diminuição de crítica. Queixam-se de pensamentos abundantes na cabeça, mostram diminuição de objetividade ou têm fugas de idéias. Podem apresentar pensamentos fantasiosos e de grandeza como o de possuir poderes mágicos, como de entender "a língua dos anjos" ou de certeza de que será bilionário. Os comportamentos bizarros e extravagantes, assim como a hipersexualização são muito mais evidentes na adolescência. ${ }^{7-}$ ${ }_{11,23}$ Carlson destaca que a irritabilidade e a labilidade emocional ocorrem mais freqüentemente em crianças menores do que nove anos, enquanto que euforia, exaltação, paranóia e delírios de grandeza são relatados com maior freqüência em crianças maiores. ${ }^{24}$ Apesar de significante, a diminuição da necessidade do sono não é mais considerada essencial para o diagnóstico de (hipo)mania em crianças para alguns pesquisadores. ${ }^{25}$

Tanto crianças como adolescentes podem ter sintomas psicóticos durante as crises de (hipo)mania. Os sintomas psicóticos mais relatados em crianças foram alucinações visuais (ex: ver cabeças voando no ar), alucinações auditivas (ex: "o diabo e o anjo estão falando comigo") e delírios de perseguição (ex: "outros meninos estão atrás de mim por inveja"). Delírios de grandeza também ocorrem (ex: ter poder de controlar o futuro).1,5,26 Geller et al encontraram $23 \%$ de crianças e adolescentes em (hipo)mania com alucinações (principalmente de comando, imperativo e religioso) e $34,6 \%$ de delírios (principalmente de grandeza, de perseguição, de referência e de culpa). ${ }^{8}$ As alucinações nesses casos são mais variadas e não se restringem em alucinações auditivas que chamam pelo nome do paciente como nos casos de depressão. A presença de sintomas psicóticos em um adulto com TAB indica pior prognóstico e maior dificuldade de tratamento, mas ainda não se definiu se o mesmo aconteceria com crianças e adolescentes. ${ }^{7-8}$

Uma outra característica do TAB-IA é o estado de humor misto. Freqüentemente encontram-se crianças ou adolescentes que apresentaram mudanças de comportamento para serem mais extravagantes, com hiperatividade psicomotora e mental, e tornam-se desinibidos socialmente, mas referindo sensações desconfortáveis de falta de esperança e que nada jamais os fariam voltar a ficar feliz. ${ }^{27}$ Como o estado de humor misto não é comum em adultos, este estado acaba sendo uma característica de crianças e adolescentes com TAB-IA. ${ }^{27}$

Outra característica é que crianças e adolescentes freqüentemente apresentam mudanças de estado de humor mais rápidas do que os adultos e podem até alternar estado de depressão e de (hipo)mania várias vezes em um mesmo dia. ${ }^{28}$ Findling et al notaram que a maioria das crianças e dos adolescentes com TAB apresentam curso tipo ciclagem ultra-rápida ou contínuo (50\%) sem período de eutimia entre os episódios. ${ }^{7}$ Geller et al destacam que, além da presença obrigatória de humor exaltado ou eufórico ou grandiosidade nas crises de (hipo)mania no caso de TAB-IA, a duração da crise de (hipo)mania pode ser variada, e chamam atenção para a presença de ciclos ultra-rápido ou contínuos em crianças, sintomas esses que eram pouco identificados anteriormente. Uma das crianças com diagnóstico de TAB tipo-II estudadas por Geller et al chegou a apresentar 104 episódios de +/- 4 horas de duração em 104 dias diferentes. ${ }^{28}$

A distinção entre estado de humor misto e ciclos ultra-rápidos ou contínuos em TAB de início precoce nem sempre é fácil. Leibenluft et al defendem que as crises de (hipo)mania com duração de 4 horas devem ser melhor investigadas para não tomar como mudança de fase as oscilações dentro de um mesmo estado de humor (ex: depressão e eutimia) e não confundir o estado de humor misto, a coexistência simultânea de sintomas de euforia e de depressão no mesmo período, com padrão de ciclagem contínuo. ${ }^{25}$

Em 2003, Leibenluft et al sugeriram definir três tipos clínicos de TAB-IA ${ }^{25}$. O primeiro é o tipo bem definido ("narrow") que é de casos que preenchem totalmente os critérios de DSM-IV para $\mathrm{TAB}$, com presença obrigatória de humor eufórico ou exaltado ou grandiosidade. Os episódios devem ter duração exigida pelo DSM-IV e ter bem definido o início e o fim de cada fase. Não se deve confundir os comportamentos pré-existentes com os sintomas de TAB. Esse tipo clínico é o tipo-I ou tipo-II do DSM-IV. ${ }^{13,25}$

O segundo é o tipo intermediário, que são de casos que apresentam sintomas de euforia, exaltação ou grandiosidade e outros sintomas de hipo(mania), cujos episódios geralmente têm duração mais curta do que exigido por DSM-IV para crise de (hipo)mania. O outro tipo intermediário é de casos que não apresentam humor eufórico e nem tem grandiosidade, mas apresentam aumento de irritabilidade e outros sintomas de (hipo)mania e têm duração prolongada. ${ }^{25}$ Os tipos intermediários geralmente recebem o diagnóstico de (hipo)mania não especificado (TAB-NOS).

O terceiro é o tipo pouco definido ("broad"), que é o daquelas crianças constantemente mal-humoradas, chateadas ou entristecidas, e que têm explosões de hiper-reatividade por estímulos emocionais negativos. Apresentam hiperatividade motora, distratibilidade, insônia, pensamento acelerado e pressão na fala e, três ou mais vezes de crises de raiva incontroláveis por semana nas últimas quatro semanas. Essas crianças têm problemas crônicos de relacionamento com pais, colegas ou irmãos que contribuem muito para prejuízo de funcionamento global dessas. ${ }^{25}$

A maioria das crianças tem estressores crônicos no ambiente familiar e social. ${ }^{7} 0$ mais recente estudo de Craney \& Geller chama atenção para o achado de que o grau de acolhimento materno pode ser um fator preditivo de recidiva após remissão, achado compatível com os estudos relativos à emoção expressa que prediz a recidiva de adultos portadores de esquizofrenia e TAB. ${ }^{9,11}$ A exposição de demais fatores psicossociais e psicodinâmicos de TAB-IA estão reservados para outros textos.

\section{Comorbidades e diagnóstico diferencial}

São relatados altos índices de comorbidade de TAB-IA com transtorno de déficit de atenção e hiperatividade (TDAH), ${ }^{29-30}$ transtorno de conduta (TC), ${ }^{31-32}$ transtornos de ansiedade, síndrome 
de pânico ou fobia social ou agorafobia ${ }^{33}$ e dependência ou abuso de substâncias psicoativas. ${ }^{32}$ Além de alto índice de comorbidades, TAB-IA também faz diagnóstico diferencial com TDAH, TC e esquizofrenia de início precoce..$^{1,34}$

As dificuldades de distinguir as manifestações clínicas de (hipo)mania de início precoce com as de TDAH e as de TC já são conhecidas. Geralmente, ou as crianças com TAB-IA são erroneamente diagnosticadas como portadoras de TDAH e TC, ou casos graves de TDAH e TC são diagnosticados como TAB-IA. , $23,29-^{-}$ 31 Sintomas presentes em transtornos comportamentais com disfunção social como hiperatividade, agressividade e infração de regras sociais também podem estar presente em crianças e adolescentes com TAB-IA. ${ }^{8-9,11,28}$

Faraone et $\mathrm{al}^{35}$ sugerem que comorbidade de TAB e TDAH pode ser uma característica de TAB-IA. Biederman et al concluíram que TAB-IA de TDAH são duas patologias distintas, mas apontam que os casos de TDAH que apresentaram posteriormente TAB-IA eram casos que desde o início tinham alto índice de comorbidades, piores escores nas entrevistas de diagnóstico $(\mathrm{CBCL})$ e história familiar de transtornos afetivos, ${ }^{29}$ ou seja, eram casos de TDAH graves. Kim e Miklowitz acham que a mesma distinção pode ser feita entre TAB e TC e, ressaltaram a possibilidade de sintomas de (hipo)mania serem indicativos de gravidade de psicopatologia na infância e não necessariamente de TAB-IA. ${ }^{31}$

Até o presente momento, o que ainda não está claro é se as comorbidades seriam verdadeiras comorbidades ou sintomas prodrômicos de TAB-IA. O grupo de pesquisadores formado por Bierderman, Wozniak e Faraone indica a possibilidade de um subtipo de TAB sempre associado a sintomas de TDAH ou até uma entidade nosográfica distinta com ocorrência simultânea de sintomas de TAB e de TDAH em criança. ${ }^{36} 0$ mesmo pode ocorrer com casos de ocorrência simultânea de TAB e TC. ${ }^{36}$ Estudos com crianças consideradas de alto risco para desenvolverem TAB, TDAH ou TC, como filhos de adultos portadores de TAB, TDAH e TC podem ser uma via para esclarecer essa questão. ${ }^{29-30,37-38}$

Para evitar confusão e dificuldade de distinguir TAB-IA e TDAH, Geller et al valorizaram apenas sintomas principais de (hipo)mania como euforia, expansão do humor ou grandiosidade para diagnóstico de TAB-IA. Os sintomas de irritabilidade e hiperatividade foram classificados como muito inespecíficos para diferenciar os dois diagnósticos. ${ }^{8-11}$ Crianças com TAB-IA apresentam maior comprometimento de humor e suas atividades tendem a ser mais direcionadas do que crianças somente com TDAH. A ocorrência de sintomas psicóticos em alguns casos de (hipo)mania e ausentes nos casos de TDAH também auxilia na distinção. ${ }^{15}$ Pesquisadores também chamam atenção para a distinção entre a real diminuição de necessidade de sono ou insônia induzida por antidepressivos nos casos de TAB com a insônia inicial ou diminuição crônica de necessidade de sono vistos em casos de TDAH. ${ }^{25}$

Alucinações incongruentes com o humor, paranóia e desorganização do pensamento são sintomas que levam à freqüente confusão de diagnóstico de TAB-IA com o de esquizofrenia. ${ }^{23,26,34,39}$ McGlashan, ao comparar pacientes com TBP de início na idade adulta com pacientes com TAB-IA, verificou que entre os casos de início precoce, $83 \%$ preenchiam critérios para transtorno esquizoafetivo no início da doença. ${ }^{39}$

\section{Tratamento}

Tradicionalmente, o tratamento de TAB em crianças e adolescentes sempre se espelhou no que já é definido para adultos. A comunidade científica tem mostrado preocupação e promovido discussões para analisar sobre: que tipo de tratamento deveria ser priorizado? Por quando tempo (duração)? Qual seria o im- pacto de tratamento precoce no curso da doença? Ainda não há respostas definitivas para essas perguntas. ${ }^{12} \mathrm{O}$ consenso atual é de que, uma vez definido o diagnóstico, o tratamento já estabelecido para o TAB deve ser prescrito imediatamente. Isso inclui introdução de estabilizador de humor, uso de antidepressivos ou de antipsicóticos - se necessário nas fases de depressão ou de (hipo)mania -, psicoterapia, orientação familiar, suporte psicopedagógico e reabilitação neuropsicológica se necessário.

Antes de iniciar o tratamento psicofarmacológico em crianças e adolescentes são obrigatórias avaliações clínicas e laboratoriais com objetivo de afastar a possibilidade de condições físicas disfuncionais (ex: disfunções hormonais) e, também, de traçar um perfil basal para futuros exames periódicos de controle. Recomenda-se avaliação hematológica, função hepática, função tireoidiana, função renal, aspectos metabólicos e ECG. A dosagem sérica de estabilizadores de humor, de anticonvulsivantes e de antidepressivos, principalmente os tricíclicos, deve ser verificada sempre que possível. ${ }^{23}$

O tratamento de depressão bipolar em crianças e adolescentes segue as mesmas regras estabelecidas para adultos. Utilizam-se os mesmos medicamentos prescritos para o adulto, mas lembrando que a maioria não passou por estudos controlados em crianças e adolescentes e alguns são até contra-indicados no momento. Deve-se lembrar que é desaconselhável o uso isolado de antidepressivo em crianças com histórico familiar de transtorno afetivo ou na presença de qualquer dos fatores de riscos citados anteriormente; aconselha-se associá-lo a um estabilizador de humor.

Ainda são raros os estudos controlados de medicação em crianças e adolescentes com TAB. Em caso de monoterapia com estabilizador de humor, há apenas um estudo controlado realizado por Geller et al utilizando lítio para adolescentes portadores de TAB e comorbidade com abuso de substância. O lítio mostrou-se eficiente na melhora das duas patologias. ${ }^{40} \mathrm{Em}$ ensaios clínicos abertos, lítio, divalproato de sódio e carbamazepina já têm mostrado isoladamente sua eficiência em casos de TAB-IA. ${ }^{26,38,41}$ Também já foram publicados alguns ensaios clínicos aberto com olanzapina ${ }^{42}$ e risperidona ${ }^{43}$ mostrando eficiência em monoterapia destas na fase aguda de (hipo)mania e manutenção de TAB-IA.

A eficácia de novos anticonvulsivantes como gabapentina, topiramato e lamotrigina na fase aguda de (hipo)mania e na manutenção de TAB ainda não foi bem esclarecida para casos de TAB-IA. A prescrição destas drogas em casos de TAB-IA espelha-se nos casos de adultos e aconselha-se usar após tentativas terapêuticas ineficientes ou intoleráveis com lítio, divalproato de sódio ou carbamazepina. ${ }^{38,44-45}$ Estudos recentes têm indicado a possibilidade e a necessidade de uso concomitante de dois estabilizadores para controle e manutenção de TAB-IA. ${ }^{38,42-43}$ Até a presente data, as pesquisas têm mostrado que o tipo de tratamento realizado parece não predizer a evolução da doença. ${ }^{11}$

Em casos de politerapia já surgiram estudos duplo-cegos controlados que fixam uso de uma droga, geralmente estabilizador de humor ou anticonvulsivante e fazem associação desta com uma outra, geralmente um antipsicótico atípico ou placebo. Foram considerados eficientes para controle de crises de TAB-IA as associações de divalproato de sódio com risperidona, de divalproato com quetiapina, de lítio e antipsicóticos atípicos e de lítio com anticonvulsivantes. ${ }^{38,42-43}$

Um estudo recente realizado nos Estados Unidos verificou que crianças e adolescentes que receberam diagnóstico de TAB geralmente são tratados com valproato de sódio (79\%), lítio (51\%) e gabapentina (29\%). Outros medicamentos utilizados foram carbamazepina (21\%), topiramato (14\%), oxacarbamazepina (6\%) e lamotrigina (4\%). Os pacientes geralmente recebem de três a cinco tipos de medicação simultaneamente e, em geral, já 
tiveram tentativas anteriores com outros seis a dez tipos de medicamentos. ${ }^{43}$ Não há trabalhos semelhantes realizados no nosso meio.

Há também cada vez mais relatos de monoterapia com antipsicóticos atípicos, como olanzapina e risperidona, para controle na fase aguda e também de manutenção de TAB-IA. ${ }^{38} \mathrm{~A}$ maioria das crianças do estudo de Bhangoo et al (77\%) já tinham tido tentativas de tratamento com antipsicóticos, mas os autores não sabem se pela presença de sintomas psicóticos nas crises ou por influência de estudos recentes que sugerem propriedades de estabilizador de humor. ${ }^{42-43}$

Causou estranheza a Bhangoo et al e preocupação com a abundância de medicamentos prescritos por médicos de comunidade, aparentemente sem sistematização e sem orientação. $15 \%$ dos casos que nunca haviam tentado tratamento com lítio, mas já haviam utilizado anticonvulsivantes novos como gabapentina, topiramato ou lamotrigina. ${ }^{43}$ Geller e Findling et $\mathrm{al}^{10,7}$ também chamaram atenção para a precariedade de diagnóstico e tratamento de TAB-IA realizado por médicos das comunidades dos Estados Unidos. Sem treinamento específico para reconhecer sintomas de (hipo)mania em crianças, estes acabam por subdiagnosticar ou superdiagnosticar e expor pacientes ao risco de prolongar o sofrimento e de tratamentos ineficientes. Note-se que esta poderia ser a situação atual do nosso Brasil.

Os aspectos etiológicos, achados neuropsicológicos e de neuroimagem serão apresentados em outros textos.

\section{Conclusões}

Muitas questões são pendentes ainda em relação a TAB-IA e algumas delas parecem ser mais incômodas do que as outras. Questões como quão precoce o diagnóstico de TAB poderia ser feito? Ou qual é o valor preditivo de manifestações precoces de TAB-IA em crianças e em adolescentes? Ou caso "sinais prodrômicos ou preditivos" fossem encontrados, qual seria o risco e o benefício de tratar as crianças e adolescentes com sintomas prodrômicos? As respostas continuam indefinidas.

\section{Referências}

1. Faedda GL, Baldessarini RJ, Suppes T, Tondo L, Becker I, Lipschitz DS. Pediatric - onset bipolar disorder; a neglected clinical and public health problem. Harv Rev Psychiatry. 1995;3(4):171-95.

2. Akiskal HS. Developmental pathways to bipolarity: are juvenile-onset depressions pre-bipolar? J Am Acad Child Adolesc Psychiatry. 1995;34(6): 754-63. Review.

3. Sanchez L, Hagino O, Weller E, Weller R. Bipolarity in children. Psychiatr Clin North Am. 1999;22(3):629-48. Review.

4. Harrington R, Myatt T. Is preadolescent mania the same condition as adult mania? A British perspective. Biol Psychiatry. 2003;53(11):961-9. Review. 5. WozniaK J. Pediatric bipolar disorder: The new perspective on severe mood dysfunction in children. J Child Adolesc Psychopharmacol. 2003; 13(4):449-51.

6. Carlson GA. Identifying prepubertal mania. J Am Acad Child Adolesc Psychiatry. 1995;34(6): 750-3.

7. Findling RL, Gracious BL, McNamara NK, Youngstrom EA, Demeter $C A$, Branicky LA, Calabrese JR. Rapid, continuous cycling and psychiatric co-morbidity in pediatric bipolar I disorder. Bipolar Disord. 2001;3(4):202-10.

8. Geller B, Zimerman B, Williams M, Bolhofner K, Craney JL, Delbello MP, Soutullo CA. Diagnostic characteristics of 93 cases of a prepubertal and early adolescent bipolar disorder phenotype by gender, puberty and comorbid attention deficit hyperactivity disorder. I Child Adolesc Psychopharmacol. 2000;10(3): 157-64.

9. Geller B, Zimerman B, Williams M, Delbello MP, Frazier J, Beringer L. Phenomenology of prepubertal and early adolescent bipolar disorder: examples of elated mood, grandiose behaviors, decreased neeed for sleep, racing thoughts and hypersexuality. J Child Adolesc Psychopharmacology. 2002; 12(1):3-9.

10. Geller B, Craney JL, Bolhofner K, Nickelsburg MJ, Williams M, Zimerman B. Two-year prospective follow-up of children with a prepubertal and early adolescent bipolar disorder phenotype. Am J Psychiatry. 2002; 159(6):927-33.

11. Craney J, Geller BA prepubertal and early adolescent bipolar disorder - I phenotype: review of phenomenology and longitudinal course. Bipolar Disord. 2003;5(4):243-56. Review.

12. National Institute of Mental Health research roundtable on prepubertal bipolar disorder. J Am Acad Child Adolesc Psychiatry. 2001;40(8):8718. Review.

13. Diagnostic and statistical manual of mental disorders: dsm-iv. $4^{a}$ ed. Washington: Apa; 1994.

14. Organização Mundial da Saúde. Classificação de transtornos mentais e de comportamento da CID-10: descrições clínicas e diretrizes diagnósticas. (trad.) Caetano D. Porto Alegre: Artes Médicas; 1993.

15. Fu-I L. Epidemiologia e fatores clínicos dos transtornos afetivos na infância e adolescência. In: Assumpção Junior B, editor. Transtornos afetivos da infância e adolescência. São Paulo: Lemos Editorial; 1996. p.19-32.

16. Kaufman J, Birmaher B, Brent D, Rao U, Flynn C, Moreci P, et al. Schedule for Affective Disorders and Schizophrenia for School-Age Children-Present and Lifetime Version (K-SADS-PL): inicial reliability and validity data. J Am Acad Child Adolesc Psychiatry. 1997;36(7):980-8. 17. Geller B, Zimerman B, Williams M, Bolhofner K, Craney JL, DelBello MP, Soutullo C. Reliability of the Washington University in St. Louis Kiddie Schedule for Affective Disorders and Schizophrenia (WASH-U-KSADS) mania and rapid cycling sections. J Am Acad Child Adolesc Psychiatry. 2001;40(4):450-5.

18. Brasil HHA. Desenvolvimento da versão brasileira da K-SADS-PL (Schedule for Affective Disorders and Schizophrenia for School Aged Children Present and Lifetime Version) e estudo de suas propriedades psicométricas [tese de doutorado]. São Paulo: Universidade Federal de São Paulo. Escola Paulista de Medicina.Psiquiatria. 2003.

19. Achenbach TM. Integrative guide for the $1991 \mathrm{cbcl} / 4-18$, ysr, and trf profiles. Burlington: University of Vermont; 1991.

20. Mick E, Biederman J, Pandina G, Faraone SV. A preliminary metaanalysis of the child behavior checklist in pediatric bipolar disorder. Biol Psychiatry. 2003;53(11):1021-7.

21. Bordin IAS, Mari JJ, Caeiro MF. Validação da versão brasileira do "Child Behavior Checklist" (CBCL) (Inventário de Comportamentos da Infância e Adolescência): dados preliminares. Rev ABP-APAL. 1995; 17(2):55-66.

22. Geller B, Fox LW, Clark KA. Rate and predictors of prepubertal bipolarity during follow-up of 6- to 12 - year-old depressed children. J Am Acad Child Adolesc Psychiatry. 1994;33(4):461-8.

23. I FL. Transtornos afetivos. Rev Bras Psiquiatr. 2000;22 Supl 2:SII24-SII27. 24. Carlson GA. Child and adolescent mania - diagnostic considerations. J Child Psychol Psychiatry. 1990;31(3):331-41. Review.

25. Leibenluft E, Charney DS, Towbin KE, Bhangoo RK, Pine DS. Defining clinical phenotypes of juvenile mania. Am J Psychiatry. 2003; 160(3):430-7. Review.

26. Varanka TM, Weller RA, Weller EB, Fristad MA. Lithium treatment of manic episodes with psychotic features in prepubertal children. Am J Psychiatry. 1988; 145(12): 1557-9.

27. Papollos D, Papollos J. Voices from the Front. In: Papollos D \& Pappolos J, editors. The Bipolar Child. New York: Broadway Books; 2002. p. 4-54. 28. Geller B, Sun K, Zimerman B, Luby J, Frazier J, Williams M. Complex and rapid-cycling in bipolar children and adolescents: a preliminary study. J Affect Disord. 1995;34(4):259-68.

29. Biederman J, Klein RG, Pine DS, Klein DF. Resolved: mania is mistaken for ADHD in prepubertal children. J Am Acad Child Adolesc Psychiatry. 1998;37(10): 1091-9.

30. Geller B, Williams M, Zimerman B, Frazier J, Beringer L, Warner KL. Prepubertal and early adolescent bipolarity differentiate from ADHD by manic symptoms, grandiose delusions, ultra-rapid or ultradian cycling. J Affect Disord. 1998;51(2):81-91.

31. Kim EY, Miklowitz DJ. Childhood mania, attention deficit hyperactivity disorder and conduct disorder: a critical review of diagnostic dilemmas. Bipolar Disord. 2002;4(4):215-25. Review.

32. Bierdeman J, Mick E, Wozniak J, Monuteaux MC, Galdo M, Faraone SV. Can a subtype of conduct disorder linked to bipolar disorder be 
identified? Integration of findings from the Massachusetts General Hospital Pediatric Psychopharmacology Research Program. Biol Psychiatry. 2003;53(11):952-60. Review.

33. Birmaher B, Kennah A, Brent D, Ehmann M, Bridge J, Axelson D. Is bipolar disorder specifically associated with panic disorder in youths? J Clin Psychiatry. 2002;63(5):414-9.

34. Carlson GA, Fennig S, Bromet EJ. The confusion between bipolar disorder and schizophrenia in youth: where does it stand in the 1990s?

J Am Acad Child Adolesc Psychiatry. 1994;33(4):453-60.

35. Faraone SV, Biederman J, Wozniak J, Mundy E, Mennin D, O'Donnell

$D$. Is comorbidity with $A D H D$ a marker for juvenile-onset mania? J Am Acad Child Adolesc Psychiatry. 1997;36(8):1046-55.

36. Wozniak J, Biederman J, Faraone SV, Blier H, Monuteaux MC. Heterogeneity of childhood conduct disorder: further evidence of a subtype of conduct disorder linked to bipolar disorder. J Affect Disord. 2001;64(23): 121-31.

37. Chang K, Steiner $H$, Ketter T. Studies of offspring of parents with bipolar disorder. Am J Med Genet. 2003;123C(1):26-35. Review. 38. Papollos D, Papollos J. Prescrisptions for Treatment. In: Papollos D \& Pappolos J, editors. The Bipolar Child. New York: Broadway Books; 2002. p. 73-144.

39. McGlashan TH. Adolescent versus adult onset of mania. Am J Psychiatry. 1988; 145(2):221-3.

40. Geller B, Cooper TB, Sun K, Zimerman B, Frazier J, Williams M, Heath J. Double-blind and placebo-controlled study of lithium for adolescent bipolar disorders with secondary substance dependency. J Am Acad Child Adolesc Psychiatry. 1998;37(2):171-8.

41. Kowatch RA, Suppes T, Carmody TJ, Bucci JP, Hume JH, Kromelis M, et al. Effect size of lithium, divalproex sodium, and carbamazepine in children and adolescents with bipolar disorder. J Am Acad Child Adolesc Psychiatry. 2000;39(6):713-20.

42. Kowatch RA, Sethuraman G, Hume JH, Kromelis M, Weinberg WA. Combination pharmacotherapy in children and adolesents with bipolar disorder. Biol Psychiatry. 2003;53(11):978-84.

43. Bhangoo RK, Lowe CH, Myers MS, Treland J, Curran J, Towbin KE, Leibenluft E. Medication use in children and adolescents treated in the community for bipolar disorder. J Child Adolesc Psychopharmacol. 2003; 13(4):515-22.

44. Frazier JA, Biederman J, Tohen M, Feldman PD, Jacobs TG, Toma V, et al. A prospective open-label treatment trial of olanzapine monotherapy in children and adolescents with bipolar disorder. J Child Adolesc Psychopharmacol. 2001;11(3):239-50.

45. Frazier JA, Meyer MC, Biederman J, Wozniak J, Wilens TE, Spencer $T J$, et al. Risperidone treatment for juvenile bipolar disorder: a retrospective chart review. J Am Acad Child Adolesc Psychiatry. 1999;38(8):960-5.

\section{Correspondência}

Lee Fu-I

Rua Padre João Manuel 450, conj. 127

Cerqueira César - 01410-001

São Paulo, SP

Tel.: (11) 3081-8249 / (11) 9808-4118

E-mail: leefui@terra.com.br 